\title{
Sentinel Lymph Node Biopsy in Patients With Thick Primary Cutaneous Melanoma
}

\author{
Juan Carlos Rodriguez Otero ${ }^{\mathrm{a}}$, Maria Susana Dagatti ${ }^{\mathrm{b}}$, Ramon Fernandez Bussy ${ }^{\mathrm{b}}$, \\ Adriana Bergero $^{\mathrm{b}}$, Mario Gorosito ${ }^{\mathrm{b}}$, Roberto Staffieri ${ }^{\mathrm{a}}$, Roberto Villavicencio ${ }^{\mathrm{a}}$, \\ Stella Maris Batalles ${ }^{\mathrm{a}}$, Stella Maris Pezzotto ${ }^{\mathrm{b}, \mathrm{c}}$
}

\begin{abstract}
Background: The clinical value of sentinel lymph node biopsy (SLNB) in patients with thick melanoma is uncertain. The purpose of this study was to investigate the correlations between survival and lymph node status in thick melanomas.

Methods: Of a total of 736 melanoma patients registered between 2000 and 2016, 50 presented with thick melanomas $(\geq 4.0 \mathrm{~mm})$ without distant metastatic disease. All patients were examined with a whole-body magnetic resonance imaging, or computed tomography, and positron emission tomography-computed tomography depending on the incorporation of the new technology in our medical institutions. They were studied according to the following procedure: 1) preoperative determination of regional lymph node along with the estimation and localization of sentinel lymph node (SLN) (dynamic isotope lymphography); 2) intraoperative localization and SLNB (lymphatic mapping); and 3) histopathology. Patient and tumor features were collected.
\end{abstract}

Results: Mean follow-up was 40 months, and 37\% had a follow-up $\geq 5$ years. A positive SLN was identified in 28 patients $(56 \%)$. No significant difference in melanoma-specific overall survival was observed in terms of the primary tumor site. Hazard ratios (HRs) were statistically significant for SLNB-positive group and mitotic rate $(\mathrm{MR})>3 \mathrm{~mm}^{2}$, but not for presence of ulceration. Mortality risk in the SLN-positive group was almost fourfold greater than that in the SLN-negative group at any time of follow-up.

Conclusions: SLN status, along with MR, can provide valuable prognostic information in patients with thick primary cutaneous melanoma.

Keywords: Lymph node status; Overall survival; Predictor factor

Manuscript submitted January 7, 2019, accepted January 31, 2019

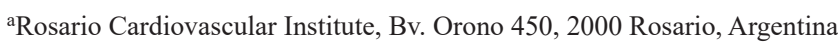
${ }^{b}$ School of Medicine, National University of Rosario, Santa Fe 3100, 2000 Rosario, Argentina

${ }^{\mathrm{c} C o r r e s p o n d i n g ~ A u t h o r: ~ S t e l l a ~ M a r i s ~ P e z z o t t o, ~ F a c u l t a d ~ d e ~ C i e n c i a s ~ M e d i c a s, ~}$ Universidad Nacional de Rosario, Santa Fe 3100, 2000 Rosario, Argentina. Email: spezzot@unr.edu.ar

doi: https://doi.org/10.14740/wjon1181

\section{Introduction}

The sentinel lymph node (SLN) technique developed by Morton et al has resulted in a significant change in the treatment approach for melanoma patients [1], and sentinel lymph node biopsy (SLNB) is generally recommended for intermediatethickness melanomas (1- $4 \mathrm{~mm})$. However, the value of this technique has recently extended to thinner lesions. The application of this technique in patients with thin $(<1 \mathrm{~mm})$ and thick $(>4 \mathrm{~mm})$ melanomas has been questioned for several reasons. While in patients with thin melanomas, questioning arises from the low probability of detecting lymph node micrometastases, while in thick melanomas, questioning arises because of the high probability of systemic spread $[2,3]$.

In the present study, our primary aim was to establish the prognostic and therapeutic value of SLN in patients with thick cutaneous melanoma (CM) determining both melanoma-specific overall survival (MSOS) and disease-free survival (DFS). Our secondary aim was to identify predictive factors for micrometastatic disease in the SLN.

\section{Materials and Methods}

We reviewed the records of all patients who had pathologically confirmed primary $\mathrm{CMs}$, with a Breslow thickness $\geq 4$ $\mathrm{mm}$, without palpable nodes or presence of distant metastatic disease or previous neoadjuvant treatments, treated between January 2000 and December 2016.

All subjects were referred to Grupo de Estudio de Melanoma Rosario (GEMRO) for surgical treatments. They underwent wide excision of the primary tumor site as potentially curative surgical therapy. GEMRO consists of health professionals belonging to Surgery, Dermatology and Pathology Department in the following institutions: Facultad de Ciencias Medicas, Universidad Nacional de Rosario, Instituto Cardiovascular de Rosario and Diagnostico Medico Orono.

The melanomas were classified and staged using the AJCC Staging System (seventh edition) [4], based on age, gender, location, histological type, Breslow thickness, Clark level, presence or absence of ulceration, mitotic rate (MR, per $\mathrm{mm}^{2}$ ), lymphovascular invasion and satellitosis. Lymph node status was also recorded.

All patients were examined with a routine whole-body 
Table 1. Patients Basic Demographics and CM Clinicopathologic Characteristics

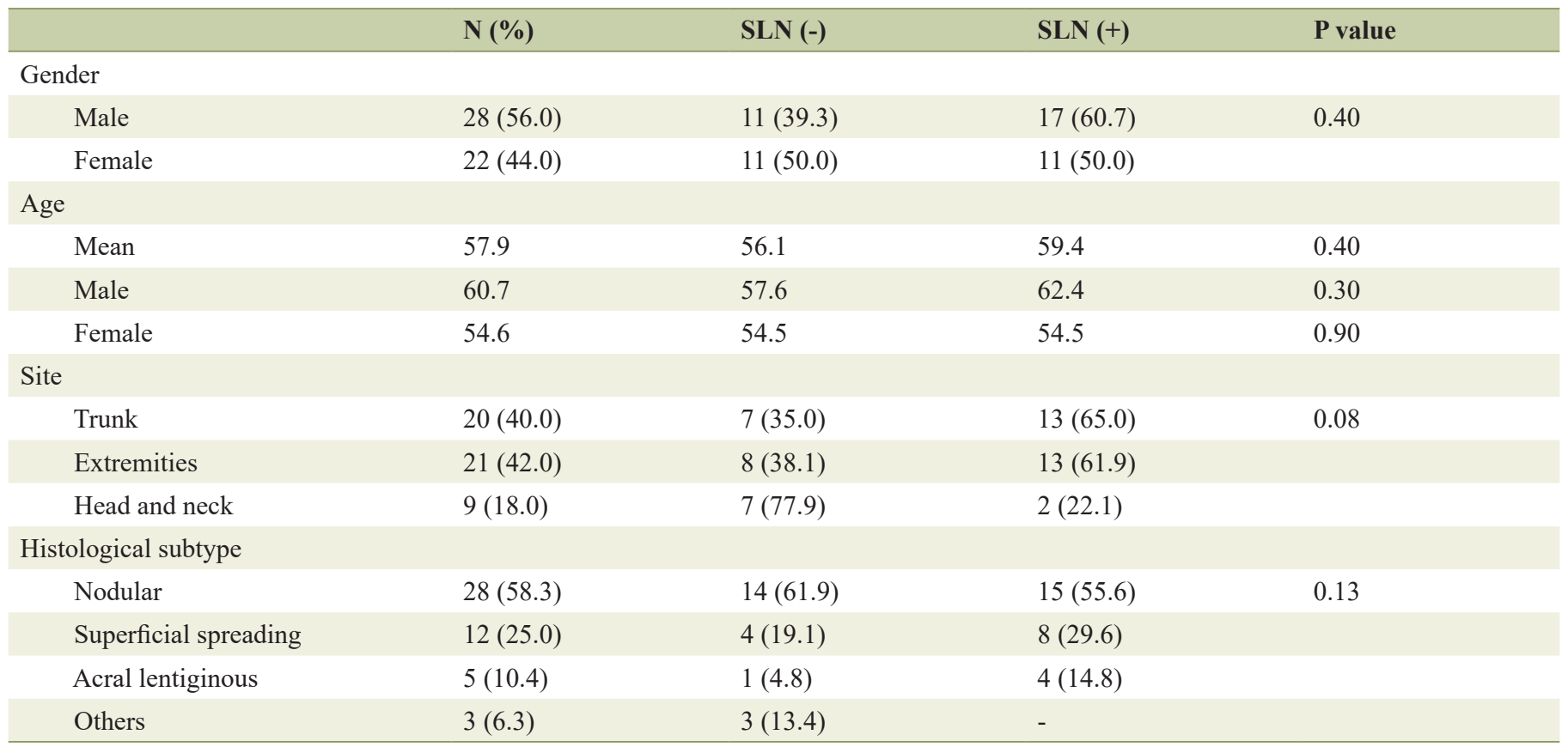

CM: cutaneous melanoma; SLN: sentinel lymph node.

magnetic resonance imaging, or computed tomography, and positron emission tomography-computed tomography depending on the incorporation of the new technology in our medical institutions.

The SLBN procedures involved the following three phases: 1) preoperative assessment of regional lymph nodes with SLN localization with a gamma probe and blue dye (dynamic isotope lymphoscintigraphy); 2) intraoperative localization and SLNB (lymphatic mapping); and 3) histopathology. Histopathological samples were stained with hematoxylin and eosin. If the result was negative, samples were studied with immunohistochemistry, as described elsewhere [5]. All previously listed factors were evaluated in association with SLN status, analyzing both MSOS and DFS.

For descriptive purposes, valid percentages (percentages based on sample size excluding missing values) were calculated. The association between SLNB results and categorical variables was compared using Chi-square test or Fisher's exact test, as appropriate. Quantitative variables were expressed as mean \pm standard deviation. The $t$-test was used to evaluate the mean differences.

The Kaplan-Meier method was used to model survival years. Stratified analyses were performed by SLN status. A log-rank (Mantel-Cox) test was used to test for differences among the strata. A similar approach was used to compare survival in the presence or absence of ulceration in each SLN stratum. Multivariate survival analysis was carried out using the Cox proportional hazards model, and covariates included were SLN status, mitoses per square millimeter and presence of ulceration. A two-tailed $\mathrm{P}$ value $<0.05$ was considered statistically significant. All data analyses were performed using Stata statistical software [6].
The study was reviewed and approved by the Ethics Committee of the School of Medicine, National University of Rosario. All the authors signed a declaration of commitment to respect the confidentiality of the data collected.

\section{Results}

From January 2000 to December 2016, 736 consecutive patients with CM aged from 22 to 88 years (mean: 49.4 years) were studied retrospectively. Among them, 50 (6.7\%) patients presented with thick melanomas without evidence of distant disease. Mean and median follow-up were 40 and 37 months, respectively (range: 12 - 120; interquartile range: 18 - 73 months); $37 \%$ of the study population had a follow-up $\geq$ 5 years. A positive SLN was identified in 28 patients $(56 \%)$. Table 1 shows the demographic and clinic pathological data from all patients according to SLN status.

Table 2 shows the SLN status and its relationship with different histopathologic parameters. Mean MR was 4, with significant differences according to SLN status $(\mathrm{P}=0.018)$. At the end of the follow-up period, 24 patients were still alive, so the MSOS was $49 \%$. The proportion of surviving patients was higher $(\mathrm{P}=0.01)$ for SLN-negative patients $(68.2 \%)$ when compared with SLN-positive patients (32.1\%). Twenty-three surviving patients were disease-free. Comparison of MSOS time between SLN-negative and SLN-positive patients revealed that mean and median survival times were shorter in the SLN-positive group (Table 3).

As shown in Figure 1, there was a significant difference in survival times between both groups $(\mathrm{P}=0.002)$.

No significant difference in MSOS was observed in terms 
Table 2. Distribution of Prognostic Factors According to SLN Status

\begin{tabular}{|c|c|c|c|c|}
\hline & Total & SLN (-) & SLN (+) & P value \\
\hline \multicolumn{5}{|l|}{ Breslow (mm) } \\
\hline Mean & 5.4 & 4.9 & 5.7 & \\
\hline \multirow[t]{2}{*}{ Range } & $4.0-12.0$ & & & \\
\hline & $4.0-5.5$ & $17(77.3 \%)$ & $18(64.3 \%)$ & 0.248 \\
\hline \multicolumn{5}{|l|}{ Ulceration } \\
\hline Presence & $36(72.0 \%)$ & $15(68.2 \%)$ & $21(75.0 \%)$ & 0.413 \\
\hline Absent & $14(28.0 \%)$ & $7(31.8 \%)$ & $7(25.0 \%)$ & \\
\hline \multicolumn{5}{|l|}{ Clark } \\
\hline III & $7(14.0 \%)$ & $3(13.6 \%)$ & $4(14.3 \%)$ & 0.510 \\
\hline$\leq 3$ & $24(49.0 \%)$ & $15(71.4 \%)$ & $9(32.1 \%)$ & \\
\hline $4-6$ & $18(36.7 \%)$ & $4(19.0 \%)$ & $14(50.0 \%)$ & 0.024 \\
\hline$>6$ & $7(14.3 \%)$ & $2(9.5 \%)$ & $5(17.9 \%)$ & \\
\hline \multicolumn{5}{|c|}{ Angiolymphatic invasion } \\
\hline Present & $12(24.0 \%)$ & $4(18.2 \%)$ & $8(28.6 \%)$ & 0.304 \\
\hline Absent & $38(76.0 \%)$ & $18(81.8 \%)$ & $20(71.4 \%)$ & \\
\hline \multicolumn{5}{|l|}{ Satellitosis } \\
\hline Present & $5(10.0 \%)$ & $3(13.6 \%)$ & $2(7.1 \%)$ & 0.384 \\
\hline Absent & $45(90.0 \%)$ & $19(86.4 \%)$ & $26(92.9 \%)$ & \\
\hline
\end{tabular}

SLN: sentinel lymph node; MR: mitotic rate.

of the primary tumor site $(\mathrm{P}=0.782)$. Hazard ratios $(\mathrm{HRs})$ according to the multivariate Cox regression method were statistically significant for SLNB (+) and MR $>3 \mathrm{~mm}^{2}$, but not for presence of ulceration. Mortality risk in the SLNB $(+)$ group was almost fourfold higher than that in the SLNB (-) group at any time of follow-up (Table 4).

\section{Discussion}

The prognosis of patients with thick melanoma is generally poor. Although some authors have questioned the usefulness of SLNB in patients with thick melanomas [7], the procedure is intended to provide prognostic and staging information.

As in other studies $[1,8,9]$, a predominance of male patients was found. In these studies, patients' mean age ranged from 58 to 69 years, and in our review, it was 57.9 years. The increased incidence of thick melanomas among older men is unexplained, but may be related to histopathologic subtype [9].

Nearly $60 \%$ of our patients had nodular melanoma histopathologic subtype. This subtype is biologically aggressive with rapid growth [10-14], and it comprised 34-69\% of melanomas $\geq 2 \mathrm{~mm}$. In our country, this histopathologic subtype represents $36.2 \%$ of all melanomas [15].

Some studies found that the most common primary tumor site is the trunk, ranged from $35 \%$ to $50 \%[9,11]$. In our pa-

Table 3. Survival Years According to SLN Status

\begin{tabular}{lllll}
\hline \multirow{2}{*}{ SLN } & \multicolumn{2}{c}{ Mean } & Median estimation \\
\cline { 2 - 5 } & Estimation & Standard error & $\mathbf{9 5 \%}$ CI & 11.17 \\
Negative & 13.98 & 2.49 & $9.10-18.87$ & 2.58 \\
Positive & 4.05 & 0.64 & $2.80-5.30$ & 5.67 \\
Global & 9.31 & 1.62 & $6.14-12.49$ & \\
\hline
\end{tabular}

SLN: sentinel lymph node; $\mathrm{Cl}$ : confidence interval. 


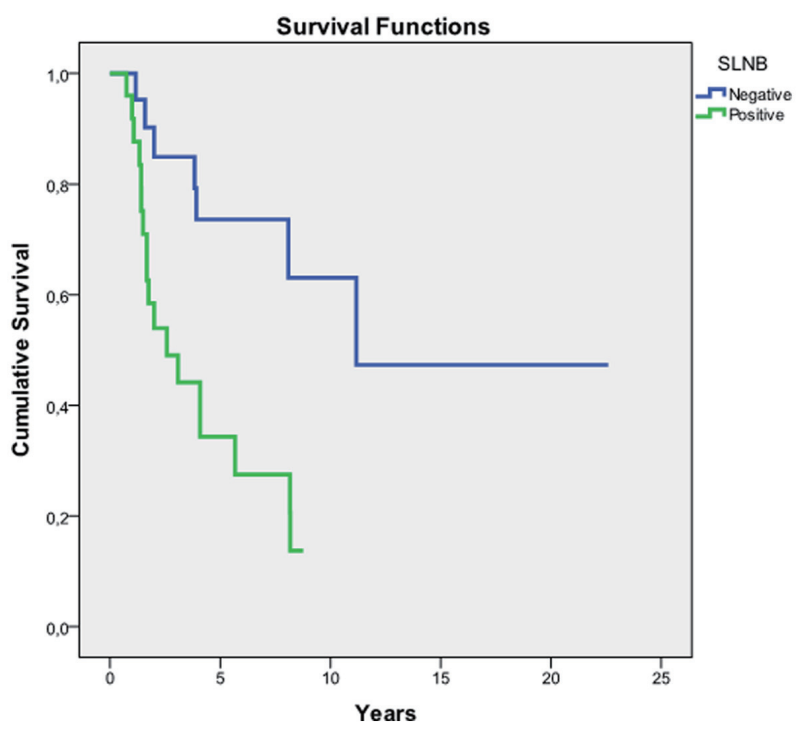

Figure 1. Kaplan-Meier estimates of survival. SLNB: sentinel lymph node biopsy; SLN: sentinel lymph node. Global comparison: test for equality of survival distributions in different levels of SLN. Log rank (Mantel-Cox) test: $\mathrm{P}=0.002$.

tients, similar figures were found for trunk and extremity primary tumor locations.

The combination of clinical and pathological characteristics in thick melanomas (i.e. nodular subtype, male sex and older age) has been seen as prognostic factors in previous studies $[10,16,17]$. However, these factors did not have a statistically significant difference in our study.

In 1970, Alexander Breslow [18] claimed that mortality from melanoma was related to thickness of the primary tumor. There is a controversy regarding the increase of thickness and its relation with the positivity of SLN $[9,11,12,15,19-21]$. It has been reported that among patients with thick melanomas $(\geq 4.0 \mathrm{~mm})$, the incidence of a positive SLN did not rise significantly with the increasing thickness [12]. A concept of thick melanomas ( $\geq 4.0$ to $<6.0 \mathrm{~mm})$ as distinct biological variants of ultra-thick melanomas $(>6.0 \mathrm{~mm})$ has been proposed; indeed a significant difference in MSOS and DFS was found between the two groups $[9,12]$. In our study, the mean tumor thickness was $5.4 \mathrm{~mm}$ (ranged from 4.0 to $12.0 \mathrm{~mm}$ ), and it was $4.9 \mathrm{~mm}$ in the SLN-negative group and $5.7 \mathrm{~mm}$ in the SLN-positive group.

Some studies have found ulceration present in $37-70 \%$ of patients $[8,22]$ and it was a prognostic factor for SLN positivity [8]. However, some authors did not find a significant prognostic impact in this subgroup of melanoma patients [12,
$19,20,23]$. In our study, an association between SLN positivity and ulceration was found in $42 \%$ of patients, whereas a negative SLN without ulceration was found in $14 \%$. This difference was not statistically significant. SLN status and ulceration were statistically significant independent predictors of survival, with SLN status being the most powerful variable [9]. Five-year overall survival for patients without ulceration and with a negative SLN was $58.8 \%$ compared to $29.4 \%$ for patients with ulceration and a positive SLN [24].

A study involving 131 patients with T4N0 primary melanomas reported that SLN status and ulceration were the most powerful indicators of DFS and overall survival, and SLNB was recommended for risk stratification in patients with thick primary melanomas [25].

It has been reported that in patients with a positive SLN, lymphovascular invasion was present in nearly a quarter of them [12], which was similar to our findings (28\%).

On multivariable analysis, a positive SLN and the presence of satellitosis were the only significant predictive factors for a worse overall survival [26]. Besides, satellitosis, lymphovascular invasion and ulceration were all significantly more likely to be associated with a positive SLN [12]. In contrast to these reports, satellitosis was present in $10 \%$ of our patients, and no significant difference in relation to SLN status was found.

MR has been reported to have a significant impact on both 5 -year DFS (46\% versus 30\%) and overall survival (74\% versus $52 \%$ ) in thick melanoma patients. Tumors with positive SLNB, high MR and thickness greater than $5.5 \mathrm{~mm}$ had significantly lower 5 -year DFS $[8,26]$. In our study, the mortality risk in patients with an MR $>3 \mathrm{~mm}^{2}$ was almost fourfold greater than in patients with a lower MR.

In our study, SLN was positive in $56 \%$ of the patients. Other studies found SLN positivity between $22 \%$ and $64 \%$ of cases $[8,9,11-15,19-21,24,27,28]$.

The presence of a positive SLN was significantly associated with an approximately $50 \%$ reduction in DFS and MSOS at both 5 and 10 years [9]. Other results indicate that SNB is the only prognostic factor of importance for survival in patients with thick melanomas [19]. Breslow thickness is known to be strongly predictive of SLN positivity, and the incidence of SLN positivity increases significantly with increasing thickness [8]. Tumors with a positive SLNB, high MR and thickness greater than $5.5 \mathrm{~mm}$ have been shown to have a significantly lower 5-year DFS [8].

We found that the SLN status is a crucial prognostic factor of greater importance than all other factors studied. In multivariate analysis, a positive SLN was the only factor decreasing MSOS. The findings that a positive SLN was an independent prognostic factor are especially relevant, since there is still

Table 4. Cox Regression Analysis for Overall Survival

\begin{tabular}{lllll}
\hline & HR $(\mathbf{9 5} \%$ CI) & P value & HR* $(\mathbf{9 5 \%}$ CI $)$ & P value \\
\hline SLNB $(+)$ & $3.8(1.5-9.6)$ & 0.005 & & 0.056 \\
MR $>3$ & $3.9(1.5-9.9)$ & 0.004 & $2.7(1.0-7.5)$ & 0.286 \\
Presence of ulceration & $2.2(0.8-6.4)$ & 0.149 & $1.8(0.6-5.3)$ & \\
\hline
\end{tabular}

HR: hazard ratio; SLNB: sentinel lymph node biopsy; Cl: confidence interval; MR: mitotic rate. *HR adjusted by SLNB status. 
controversy regarding to the value of SLNB in thick melanomas $[22,29]$.

The main limitation of this study is that its conclusions are based on retrospective, observational data. Another limitation is the small number of included patients in matched samples.

In conclusion, not all patients with thick melanoma develop distant disease. Therefore, we consider that these patients have an uncertain prognosis and we cannot predict with confidence which patients will or will not progress; for that reason, SLNB should be added to clinical observation. Even in patients with thick melanoma, a negative SLNB still predicts a better survival than a positive SLNB, as has been previously demonstrated in patients with thinner melanomas [5]. We believe that patients with thick melanomas are a heterogenous group and should be offered SLNB as part of their staging. A noteworthy observation from our study is that SLN status, along with histologic factors such as MR, can combine to provide valuable prognostic indicators for patient survival in patients with thick CM.

\section{Acknowledgments}

The authors are grateful to Professor John F. Thompson, from the Melanoma Institute Australia, for the revision of the manuscript.

\section{Funding Support}

The study was supported by National University of Rosario, Argentina.

\section{Conflict of Interest}

All authors declared no conflict of interest.

\section{Abbreviations}

SLN: sentinel lymph node; SLNB: sentinel lymph node biopsy; CM: cutaneous melanoma; MSOS: melanoma-specific overall survival; DFS: disease-free survival; GEMRO: Grupo de Estudio de Melanoma Rosario

\section{References}

1. Morton DL, Wen DR, Wong JH, Economou JS, Cagle LA, Storm FK, Foshag LJ, et al. Technical details of intraoperative lymphatic mapping for early stage melanoma. Arch Surg. 1992;127(4):392-399.

2. Warycha MA, Zakrzewski J, Ni Q, Shapiro RL, Berman RS, Pavlick AC, Polsky D, et al. Meta-analysis of sentinel lymph node positivity in thin melanoma ( $<$ or $=1 \mathrm{~mm})$. Cancer. 2009;115(4):869-879.

3. Balch CM, Gershenwald JE, Soong SJ, Thompson JF,
Atkins MB, Byrd DR, Buzaid AC, et al. Final version of 2009 AJCC melanoma staging and classification. J Clin Oncol. 2009;27(36):6199-6206.

4. Edge S, Byrd DR, Compton CC, Fritz AG, Greene FL, Trotti A. AJCC Cancer Staging Handbook. From the AJCC Cancer Staging Manual 7th ed. Springer-Verlag, New York, 2010.

5. Rodriguez Otero JC, Fernandez Bussy R, Bergero A, Gorosito $\mathrm{M}$, Salerni G, Dagatti MS, Villavicencio R, et al. Thin melanoma and sentinel lymph node biopsy: A difficult relationship between them. Integr Cancer Sci Therap. 2016;3(6):1-5.

6. StataCorp. 2005. Stata Statistical Software: Release 9. College Station, TX: Stata Corp LP.

7. Essner R, Chung MH, Bleicher R, Hsueh E, Wanek L, Morton DL. Prognostic implications of thick ( $>$ or $=4-\mathrm{mm}$ ) melanoma in the era of intraoperative lymphatic mapping and sentinel lymphadenectomy. Ann Surg Oncol. 2002;9(8):754-761.

8. White I, Fortino J, Curti B, Vetto J. Clinical impact of sentinel lymph node biopsy in patients with thick $(>4$ mm) melanomas. Am J Surg. 2014;207(5):702-707; discussion 707.

9. Meguerditchian AN, Asubonteng K, Young C, Lema B, Wilding G, Kane JM, 3rd. Thick primary melanoma has a heterogeneous tumor biology: an institutional series. World J Surg Oncol. 2011;9:40.

10. Demierre MF, Chung C, Miller DR, Geller AC. Early detection of thick melanomas in the United States: beware of the nodular subtype. Arch Dermatol. 2005;141(6):745750 .

11. Jacobs IA, Chang CK, Salti GI. Role of sentinel lymph node biopsy in patients with thick $(>4 \mathrm{~mm})$ primary melanoma. Am Surg. 2004;70(1):59-62.

12. Gajdos C, Griffith KA, Wong SL, Johnson TM, Chang AE, Cimmino VM, Lowe L, et al. Is there a benefit to sentinel lymph node biopsy in patients with T4 melanoma? Cancer. 2009;115(24):5752-5760.

13. Rughani MG, Swan MC, Adams TS, Marshall A, Asher $\mathrm{R}$, Cassell OC, Middleton MR. Sentinel node status predicts survival in thick melanomas: the Oxford perspective. Eur J Surg Oncol. 2012;38(10):936-942.

14. Fairbairn NG, Orfaniotis G, Butterworth M. Sentinel lymph node biopsy in thick malignant melanoma: a 10year single unit experience. J Plast Reconstr Aesthet Surg. 2012;65(10):1396-1402.

15. Leitner RMC. Epidemiologia del melanoma cutaneo. Rev Argent Dermatol. 2006;87:86-97.

16. Murray CS, Stockton DL, Doherty VR. Thick melanoma: the challenge persists. Br J Dermatol. 2005;152(1):104109.

17. Chamberlain AJ, Fritschi L, Giles GG, Dowling JP, Kelly JW. Nodular type and older age as the most significant associations of thick melanoma in Victoria, Australia. Arch Dermatol. 2002;138(5):609-614.

18. Breslow A. Thickness, cross-sectional areas and depth of invasion in the prognosis of cutaneous melanoma. Ann Surg. 1970;172(5):902-908.

19. Vermeeren L, van der Ent FW, Sastrowijoto PS, Hulsewe 
KW. Thick melanoma: prognostic value of positive sentinel nodes. World J Surg. 2009;33(11):2464-2468.

20. Kelly J, Redmond HP. The role of sentinel lymph node biopsy in patients with thick melanoma. A single centre experience. Surgeon. 2012;10(2):65-70.

21. Ribero S, Osella-Abate S, Sanlorenzo M, Balagna E, Senetta R, Fierro MT, Macripo G, et al. Sentinel lymph node biopsy in thick-melanoma patients $(\mathrm{N}=350)$ : What is its prognostic role? Ann Surg Oncol. 2015;22(6):19671973.

22. Robinson EM, Rosenbaum BE, Rogers R, Han SW, Tchack J, Moran U, Zhong J, et al. Prognostic value of mitoses in thick primary melanoma. J Clin Oncol. 2016;34:(15 suppl)e21046.

23. Rodriguez-Lomba E, Marquez-Rodas I, Mercader-Cidoncha E, Suarez-Fernandez R, Aviles-Izquierdo JA. Why do patients with thick melanoma have different outcomes? A retrospective epidemiological and survival analysis. Clin Transl Oncol. 2017;19(8):1055-1057.

24. Monroe MM, Myers JN, Kupferman ME. Undertreatment of thick head and neck melanomas: an age-based analysis. Ann Surg Oncol. 2013;20(13):4362-4369.

25. Gershenwald JE, Mansfield PF, Lee JE, Ross MI. Role for lymphatic mapping and sentinel lymph node biopsy in patients with thick ( $>$ or $=4 \mathrm{~mm}$ ) primary melanoma. Ann Surg Oncol. 2000;7(2):160-165.

26. Gyorki DE, Sanelli A, Herschtal A, Lazarakis S, McArthur GA, Speakman D, Spillane J, et al. Sentinel Lymph Node Biopsy in T4 Melanoma: An Important Risk-Stratification Tool. Ann Surg Oncol. 2016;23(2):579-584.

27. Morton DL, Thompson JF, Cochran AJ, Mozzillo N, Nieweg OE, Roses DF, Hoekstra HJ, et al. Final trial report of sentinel-node biopsy versus nodal observation in melanoma. N Engl J Med. 2014;370(7):599-609.

28. Yamamoto M, Fisher KJ, Wong JY, Koscso JM, Konstantinovic MA, Govsyeyev N, Messina JL, et al. Sentinel lymph node biopsy is indicated for patients with thick clinically lymph node-negative melanoma. Cancer. 2015;121(10):1628-1636.

29. Madu MF, Wouters MW, van Akkooi AC. Sentinel node biopsy in melanoma: Current controversies addressed. Eur J Surg Oncol. 2017;43(3):517-533. 